\title{
Otolaryngological manifestations of hospitalised patients with confirmed COVID-19 infection
}

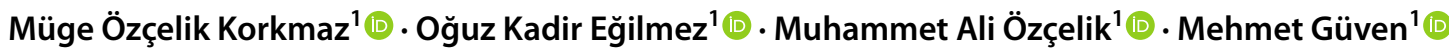

Received: 9 July 2020 / Accepted: 23 September 2020 / Published online: 3 October 2020

c) Springer-Verlag GmbH Germany, part of Springer Nature 2020

\begin{abstract}
Purpose The aim of our study is to evaluate the incidence and characteristics of otolaryngology symptoms in COVID 19 patients.

Methods 116 patients with positive PCR test results for COVID-19 and followed up by otolaryngologists at a tertiary referral center/COVID-19 pandemic hospital were questioned in terms of otolaryngology symptoms associated with COVID-19 infection. Data including demographics, disease severity, concomitant diseases, previous otolaryngologic diseases, incidence and duration of new onset symptoms were collected and categorically analyzed. In addition, the severity of loss of smell and taste was evaluated by visual analogue score (VAS).

Results A total of 58 men and 58 women participated. The mean age of the patients was $57.24 \pm 14.32$ (19-83). The most common otolaryngological findings were hyposmia/anosmia (37.9\%) and hypogeusia/ageusia (41.37\%), respectively. These complaints were followed by headache (37.1\%), and nausea/vomiting (31\%). The most common oropharyngeal symptoms were sore throat $(32.7 \%)$ and dysphagia (20.6\%). The rate of otological/vestibular symptoms was dizziness $(31.8 \%)$, tinnitus (11\%), true vertigo (6\%), and hearing impairment (5.1\%), respectively. The most of symptoms were more frequent in $>60$ years and women. There was a significant correlation between nasal itching and smell disturbance in patients with allergic rhinitis. Considering the duration of symptoms, the longest were hyposmia/anosmia and hypogeusia/ageusia. The mean VAS's in patients with hyposmia/anosmia and hypogeusia/ageusia were $5.52 \pm 2.08$ and $5.79 \pm 2.21$, respectively.

Conclusion The most common otolaryngologic symptoms of COVID-19 infection are known as sore throat, smell and/ or taste disturbances. However, our study showed that these patients can be seen with different symptoms in otological or laryngeal areas. Therefore, a more careful evaluation should be made in terms of otolaryngologic symptoms when COVID 19 infection is suspected.
\end{abstract}

Keywords COVID-19 $\cdot$ Rhinology $\cdot$ Otology $\cdot$ Vertigo $\cdot$ Throat $\cdot$ Allergic rhinitis

\section{Introduction}

The coronavirus disease 2019 (COVID-19), which is caused by severe acute respiratory syndrome coronavirus 2 (SARS$\mathrm{CoV}-2$ ), is an ongoing viral pandemic that emerged from the city of Wuhan, China and quickly spread to the rest of the world [1]. This outbreak has been responsible for nearly 935,000 deaths worldwide and more than 29 million people have been confirmed as infected so far [2]. The virus is

Müge Özçelik Korkmaz

ozcelikmuge@gmail.com

1 Sakarya University Medical Faculty Traning and Research Hospital, Otolaryngology Department, Korucuk Campus, Korucuk, 54290 Sakarya, Turkey transmitted from human to human via droplet transmission and direct contact with oral, nasal, and eye mucous membranes [3]. Studies suggest that COVID-19 may become airborne through aerosol-generating clinical procedures [4].

Coronaviruses are known to cause diseases of varying severity affecting respiratory, neurological, gastrointestinal, and hepatic organs in animals and humans [4]. The patients with the classical symptoms of COVID-19 are easily diagnosed; however, it can be difficult to recognize those without characteristic findings and pose a risk for transmission, thereby causing the disease to last longer and be persistent [5]. According to the clinical reports from Asia, the most frequently observed symptoms are cough, fever, sputum production, dyspnea, arthralgia, myalgia, rhinorrhea, sore throat, headache, and diarrhea [6, 7]. Since the virus is first 
transmitted to the body through the upper respiratory tract with droplets, it is unavoidable to encounter otolaryngologic symptoms. The above-mentioned symptoms have been reported and they are the most common findings for COVID19; however, several studies, particularly from Europe, have also reported certain chemosensory dysfunction symptoms such as anosmia and ageusia, which are common in COVID positive patients [8]. Hyposmia can generally be caused by upper respiratory tract infections; however, these symptoms can be present in COVID-19 patients without any nasal symptoms, suggesting the direct viral damage to the chemosensory system [9]. Considering the neural effects of the virus, it is likely to affect other cranial nerve pairs, including the vestibular and acoustic nerve, and can cause associated symptoms. However, there are not enough publications in the literature on this subject yet.

Because of the paucity of diagnostic tests in many European countries, data regarding epidemiological factors and clinical presentation of COVID-19 positive patients are limited; the reported studies were generally carried out by anamnesis and symptom inquiry [10]. The aim of this study is to evaluate specifically the otolaryngologic symptomatology of COVID-19 positive patients followed up in the otolaryngology clinic, to reveal the findings encountered and their frequency, and to investigate whether there are different findings in the Turkish population other than those reported in other countries.

\section{Materials and methods}

\section{Patient selection and questionnaire}

The present study is a prospective observational cohort study. The study protocol was approved by the Ethics Committee of one hundred and sixteen patients with COVID-19 infection with a positive reverse transcription-polymerase chain reaction (RT-PCR) test result, who were followed up and treated by ear-nose-throat (ENT) specialists in a tertiary and pandemic hospital between April and May 2020, were included in the study. The inclusion criteria were being between the ages of 18-90, having no mental or physical defects preventing communication, no previous history of nasal, oropharyngeal or laryngeal surgery, no serious neurological deficit, or no history of the nervous system disease. In addition, patients, who used drugs affecting the vestibular or central nervous system (e.g., sedative and hypnotic drugs such as benzodiazepines, antihistaminic drugs such as betahistine and dimenhydrinate, and anticonvulsant drugs such as phenytoin and carbamazepine), were not included in this study. An informed consent form was obtained from all patients before participating in the study.
All of the patients included in this study had positive results of the COVID -19 RT-PCR test, which was studied with nasopharyngeal/oropharyngeal swab samples. In addition, clinical findings of all patients, thoracic computed tomography (CT) and / or laboratory tests (Hemogram, Urea, Creatinine, Albumin, Alanine aminotransferase (ALT), Aspartate Aminotransferase (AST), C-reactive protein (CRP), Sedimentation, Lactate Dehydrogenase (LDH), D-Dimer, Cardiac Troponins (TnT / TnI), Creatine kinase (CK / CK-MB), albumin ferritin) were evaluated to be compatible with SARS-COV 2 infection. During the study, the patients, who were transferred to the intensive care unit due to deterioration in their health status were excluded from the study. Patients, who continued their current symptoms while discharged from hospital, were then contacted by telephone, and the duration of their symptoms was followed up. In addition to demographic data such as the age and gender of the patients, general data including the concomitant systemic diseases, previous otolaryngologic diseases (perennial/allergic rhinitis, nasal septal deviation, chronic sinusitis, nasal polyps, hearing loss, tinnitus, vestibular disorders), the use of medications, and the length of hospital stay were also recorded. In addition, clinical data (fever, pulse, arterial blood pressure, and oxygen saturation), laboratory tests, and thorax CT results were recorded. The clinical severity was evaluated based on laboratory, radiologic and clinical data. Patients were classified as mild (1) or moderate (2) according to the latest WHO joint report [11]. A questionnaire form that included the otolaryngologic and general manifestations and aimed at questioning the new-onset symptoms was filled in individually for every patient. The onset times of all available symptoms and their durations were questioned and recorded; therefore, the average times for each symptom were calculated (Supplemental questionnaire).

To evaluate the loss of sense of smell and taste, the Visual Analogue Scale (VAS) was used, taking the sample of the American Academy of Otolaryngology-Head and Neck Surgery COVID-19 Anosmia Reporting tool [12]. Individuals were asked to evaluate their ability to smell and taste scoring between 0 (I cannot smell / taste) and 10 (normal). VAS score averages of those with loss of smell/taste sensation were then calculated.

\section{Categorical evaluation}

Patients were evaluated in five different categorical groups in terms of age, gender, disease severity, concomitant systemic, and otolaryngologic diseases. They were examined in two subgroups, which were $<60$ years and $\geq 60$ years in terms of age, mild and moderate in terms of disease severity, Yes or No in terms of having a history of concomitant otolaryngologic (nasal, otologic, etc.) disorder, Yes or No in terms of concomitant systemic diseases (DM, HT, renal failure, 
cardiac diseases,respiratory diseases, others). The presence of differences in symptoms and the VAS score averages of those with loss of smell/taste sensation were compared in terms of categorical groups.

\section{Statistical analysis}

We used descriptive statistics, such as mean, standard deviation (SD), and distribution to analyze patient characteristics. The categorical data were given as numbers $(n)$ and percentages (\%). The Kolmogorov-Smirnov test was performed for the normality distribution analysis; and according to the results, nonparametric tests were conducted. Independent sample $t$ test was used for comparing the mean between groups. Comparisons between continuing data were analyzed with the Mann-Whitney test. Fisher's Exact test and Pearson Chi square tests were used for the comparison of categorical variables, where $p$ values less than 0.05 were accepted as significant. All statistical analyses were performed using commercial software (IBM SPSS Statistics. Version 23.0. Armonk. NY: IBM Corp).

\section{Results}

A total of 116 patients consisting of 58 males (50\%) and 58 females $(50 \%)$ were included in the study. The mean age of the patients was $57.24 \pm 14.32$ (min 19-max 83). The patients were grouped in terms of age, where 62 people $(53.4 \%)$ were under the age of 60,54 people $(46.6 \%)$ were in the group over 60 . There was no statistical difference in terms of gender and mean age between groups $(p>0.05$ for both). The average length of hospital stay was 14.36 6.42 days.

Thorax CT scans with unilateral or bilateral findings compatible with COVID 19 pneumonia such as consolidation, frosted glass opacities, crazy paving patterns, and linear opacities were found in $48 \%$ of the patients. While $59(50.9 \%)$ of the patients did not have any concomitant systemic diseases, 57 (19.8\%) people had one or more concominant diseases. The severity of COVID-19 infection was mild in $83(71.5 \%)$ and moderate in 33 patients $(28.4 \%)$. While $89(76.7 \%)$ patients had no previous otolaryngologic disease, $14(12.1 \%)$ had perennial/seasonal allergic rhinitis, $8(6.9 \%)$ had sinonasal problems, $3(2.5 \%)$ had hearing loss/ tinnitus, and $2(1.7 \%)$ had vestibular problems (Table 1).

Information on the frequency of symptoms is given in Fig. 1. The most common general findings were dry cough $(53.4 \%)$, dyspnea $(38.8 \%)$, headache $(37 \%)$ and nausea/ vomiting $(31 \%)$. In terms of otolaryngological symptoms these complaints were followed by hypogeusia/ageusia (41.3\%) and hyposmia/anosmia (37.9\%). Symptoms related to pharynx/larynx such as sore throat, dysphagia, voice
Table 1 Age, gender, concomitant diseases, clinic severity and previous ENT disorders information of patients who including the study

\begin{tabular}{lc}
\hline Datas & $N(\%)$ \\
\hline Age & \\
$<60$ years & $62(53.5)$ \\
$\geq 60$ years & $5(46.5)$ \\
Gender & \\
Male & $58(50)$ \\
Female & $58(50)$ \\
Concomitant diseases & \\
Hypertension & $23(19.8)$ \\
Diabetes mellitus & $13(11.2)$ \\
Renal failure & $5(4.3)$ \\
Coronary artery/cardiac disease & $7(6)$ \\
Chronic respiratory disease/Asthma & $8(6.9)$ \\
Other & $3(2.5)$ \\
Clinic & \\
Mild & $83(71.5)$ \\
Moderate & $33(28.4)$ \\
Previous ENT disease & \\
Allergic Rhinitis & $14(12.1)$ \\
Sinonasal problems & $8(6.8)$ \\
Hearing loss/tinnitus & $3(2.5)$ \\
Vestibular disorders & $2(1.7)$ \\
\hline
\end{tabular}

ENT ear-nose-throat, $N$ number of patients

impairment and globus sensation were detected in $32.7 \%$, $20.6 \%, 19.8 \%$, and $13.7 \%$, respectively. Nasal symptoms were the least often symptoms encountered by the patients. Nasal obstruction was observed in $27.5 \%$ of the patients, rhinorrhea in $13.7 \%$, and sneezing/nasal itching in $12.9 \%$. Considering the distribution of otologic / vestibular symptoms, the rates were as follows; dizziness $(31.8 \%)$, tinnitus (11.2\%), hearing impairment (5.2\%) and true vertigo (6.1\%). Ocular complaints were present in $17.2 \%$ of patients. High fever occurred in $27.5 \%$ of the patients.

The relation of symptoms in terms of age, gender, the severity of the disease, concomitant systemic diseases, previous otolaryngologic diseases (perennial/seasonal allergic rhinitis) are given in Table 2. When the patients were grouped in terms of age, it was found that many symptoms such as tinnitus, true vertigo, headache, smell/taste impairment, sore throat and voice impairment were statistically significant common in patients under 60 years of age ( $p<0.05$ for each). Dyspnea and cough were more common in patients over 60 years. In terms of gender distribution, the rate of dizziness, headache, smell/taste impairment, sore throat, and voice complaints were statistically significant higher in women. When evaluated according to the clinical severity of COVID-19 infection, there was no statistically significant difference between other findings except nausea/ 
Fig. 1 Distribution frequency of symptoms

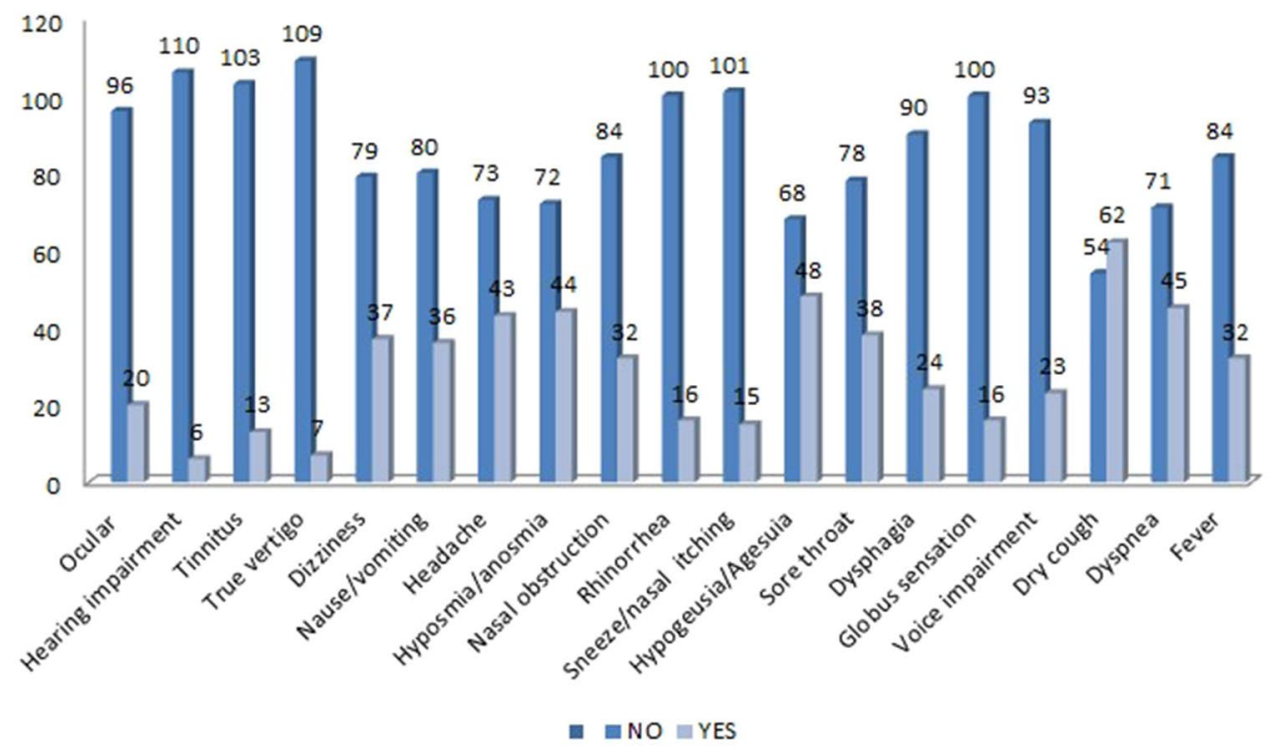

vomiting, cough and dyspnea which were higher in the moderate group. The symptoms of nasal itching and hiposmia/ anosmia were statistically significantly higher in patients with concomitant otolaryngologic diseases $(p<0.05)$. Ten of 15 patients with nasal itching and 12 of patients with smell loss had a history of seasonal allergic rhinitis. In terms of comorbidities, the incidence of headache was higher in patients with hypertension / hypertension + diabetes mellitus. Again, dyspnea was higher in patients with chronic respiratory disease. No statistically significant difference was found between patients with and without concominant systemic disease in terms of other symptoms (Table 3). When the VAS scores regarding the sense of smell and taste were examined, the mean VAS was $5.52 \pm 2.08(\min 0-\max$ 8) for those who stated hyposmia/anosmia, and $5.79 \pm 2.21$ (min 0-max8) for those who stated that they had hypogeusia/ ageusia. Accordingly, the average VAS scores in the group 60 years and older were found to be statistically significantly lower in the evaluation of the VAS averages among the categories in patients with hyposmia/anosmia and hypogeusia/ ageusia $(p<0.05)$. In other groupings, no statistically significant difference was found in terms of VAS averages.

Data on the maximum, minimum and median duration of the symptoms over a period of 30 days are shown in Fig. 2 . Accordingly, the longest-onset otolaryngologic symptom was found to be symptoms related to taste and smell. There was no statistically significant difference between the other symptom duration averages.

To identify the questions that tend to be correlated, factor analysis was used with the assumption of the correlation between factors or subgroups of questions. In Fig. 3, the results are shown only for factors with eigen values greater than one. It was found that symptoms were loaded in six different factorial subgroups. The complaints about hearing, tinnitus and vertigo constituted the first subgroup (otological); difficulty in swallowing, sore throat, globus sensation and voice impairment constituted the second (pharyngeal); smell/taste impairment symptoms constituted the third (smell and taste); nasal congestion, runny nose, and sneezing constituted the fourth (nasal); dizziness, nausea, headache constituted the fifth (neurovegetative); and fever, cough, and dyspnea constituted the sixth (others) subgroup.

\section{Discussion}

The main transmission route for SARS-Cov-2 is known as airborne droplets In addition, the virus can also be transmitted by touching hands to the mouth, nose, or eye mucosa after contact of droplets made by coughs and sneezes from sick people. Therefore, it is very likely that the disease will show signs and symptoms in the eyes and upper respiratory tract, which is the first entry point into the body. In some studies, conjunctivitis has also been described [13, 14]. This present study, it was found that PCR positive COVID 19 patients had different otolaryngological symptoms, especially loss of smell and taste.

Symptomatic patients can range from a mild to a heavy clinical status; however, in the vast majority of patients, the situation is not very severe. In a study evaluating the disease severity of approximately 44,500 definitive cases from the China Disease Control and Prevention Center, $81 \%$ of the patients had mild (no pneumonia or mild pneumonia), $14 \%$ had severe (e.g., dyspnea, hypoxia, or developing more than $50 \%$ lung involvement in imaging within $24-48 \mathrm{~h}$ ) and 5\% critical disease (e.g., respiratory failure, shock or multiorgan dysfunction) have been reported [15]. Since the health status of the patients, who were followed up in the clinic, was in 


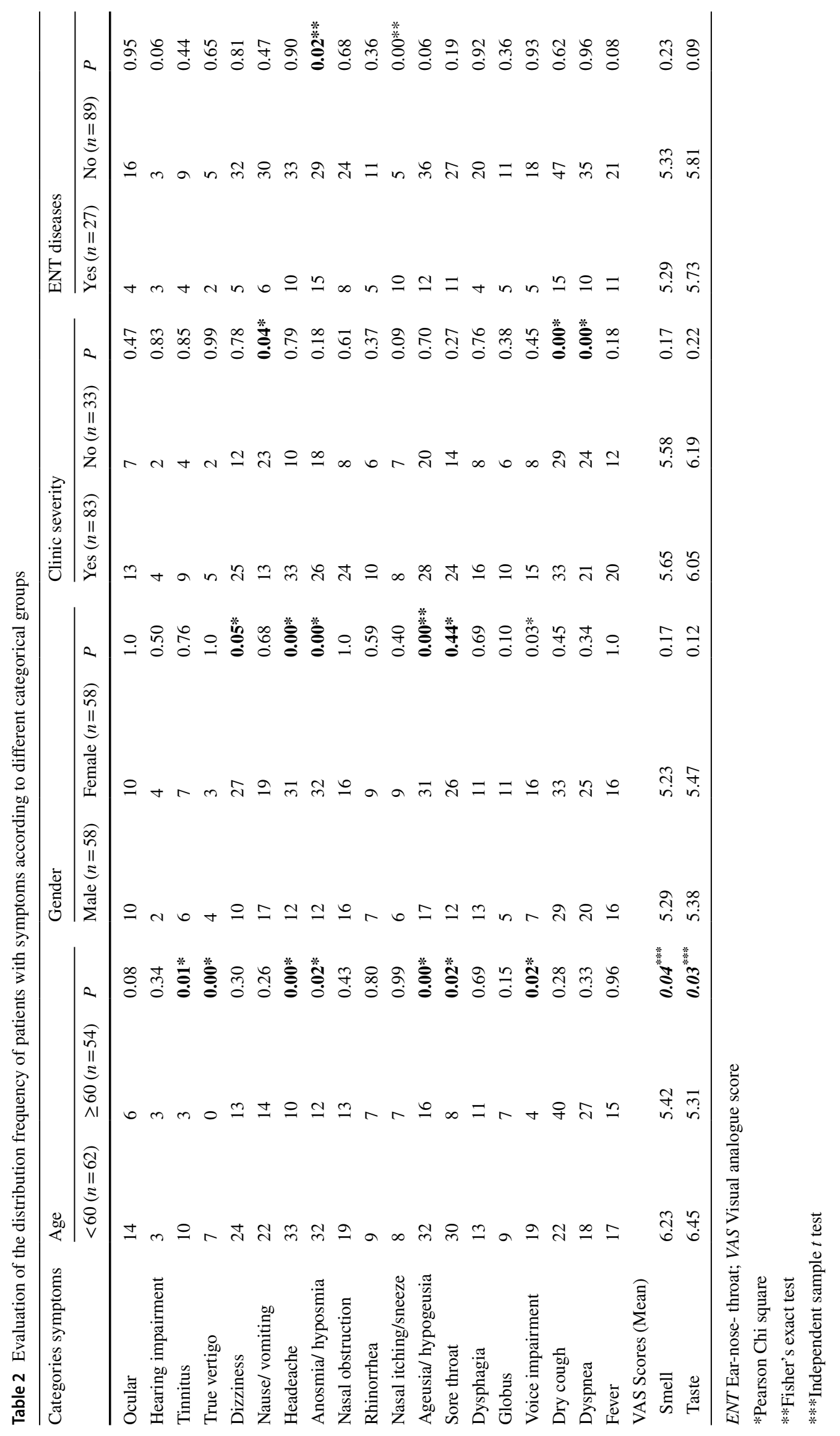




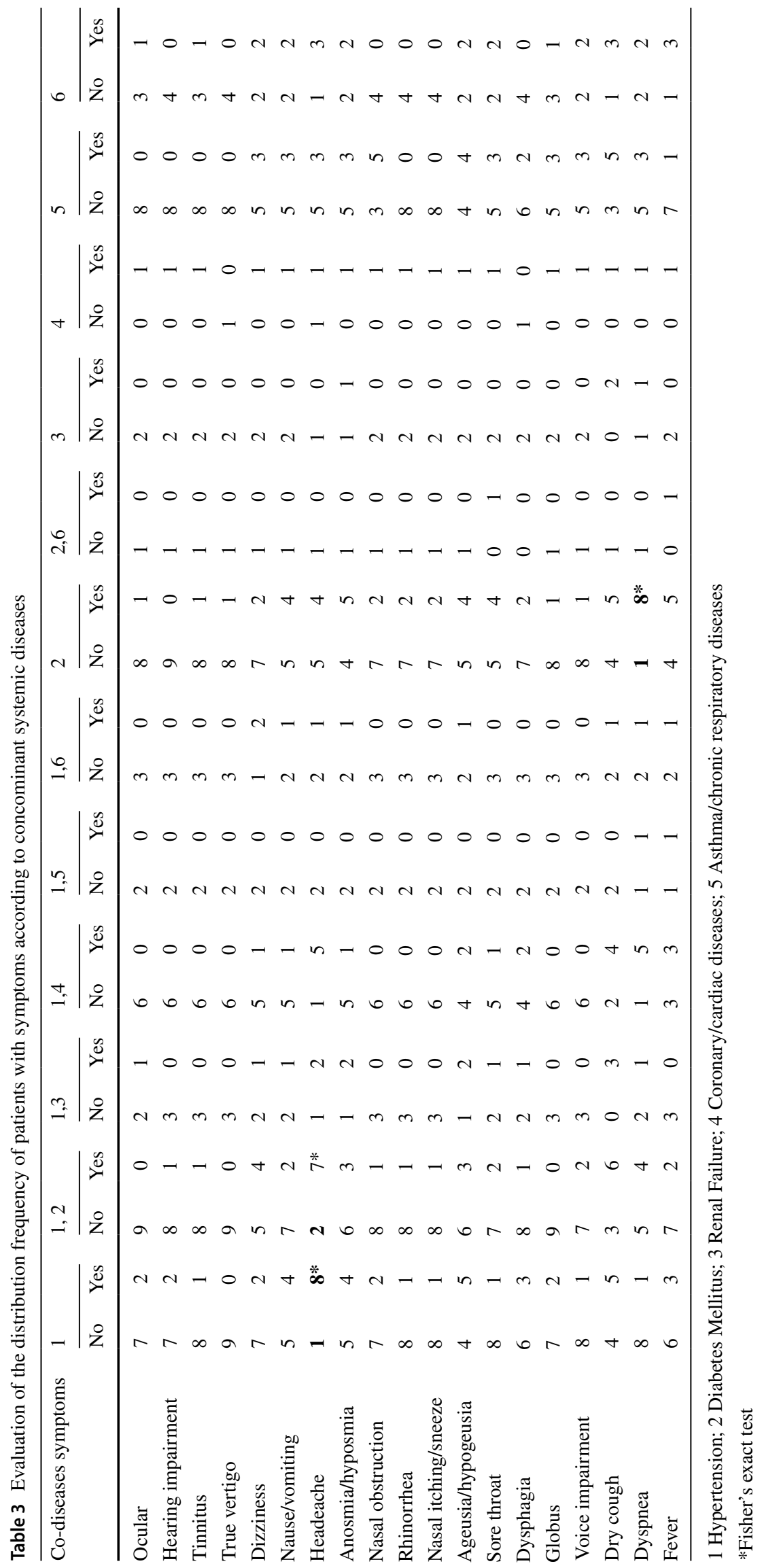


Fig. 2 Data on the maximum, minimum and median duration of the symptoms over a period of 30 days

Fig. 3 Factor analysis with Promax rotation of the frequencies for the 19 questions related to otolaryngology in COVID 19 patients. The questions are divided into six different subgroups in relation to each other

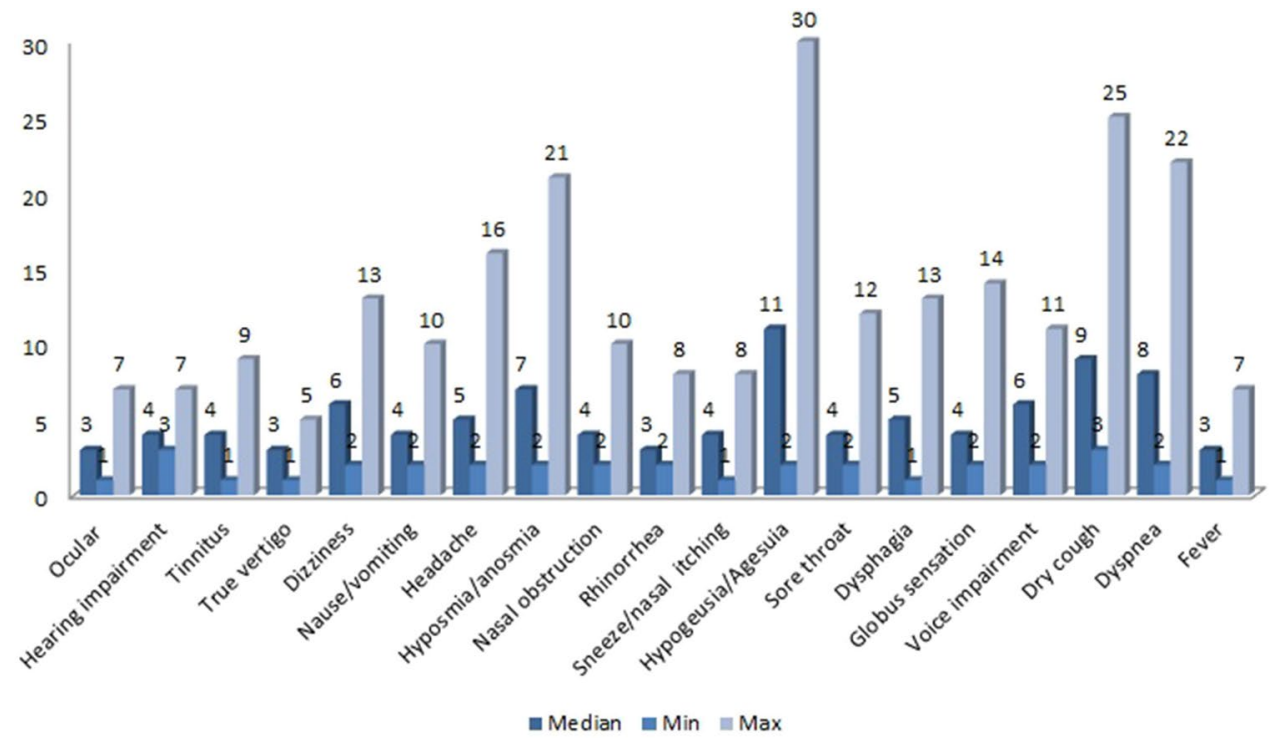

Pattern Matrix ${ }^{a}$

\begin{tabular}{|c|c|c|c|c|c|c|}
\hline & \multicolumn{6}{|c|}{ Component } \\
\hline & 1 & 2 & 3 & 4 & 5 & 6 \\
\hline $\begin{array}{l}\text { Hearing } \\
\text { impairment }\end{array}$ & 0,817 & & & & & \\
\hline Tinnitus & 0,726 & & & & & \\
\hline True vertigo & 0,661 & & & & & \\
\hline Ocular & & & & & & \\
\hline Dysphagia & & $-0,851$ & & & & \\
\hline Sore throat & & $-0,744$ & & & & \\
\hline Globus sensation & & $-0,648$ & & & & \\
\hline Smell & & & 0,807 & & & \\
\hline Taste & & & 0,636 & & & \\
\hline Nasal obstruction & & & 0,503 & $-0,400$ & & \\
\hline Rhinorrhea & & & & $-0,826$ & & \\
\hline Itching/sneeze & & & & $-0,813$ & & \\
\hline Dizziness & & & & & $-0,708$ & \\
\hline Nause/vomiting & & & & & $-0,703$ & \\
\hline Headeache & & & & & $-0,438$ & \\
\hline Voice impairment & & $-0,386$ & & & $-0,388$ & \\
\hline Fever & & & & & & 0,743 \\
\hline Dyspnea & & & & & & 0,654 \\
\hline Cough & & & & & & 0,423 \\
\hline
\end{tabular}

Extraction Method: Principal Component Analysis.

a. Rotation converged in 14 iterations. 
the mild and moderate level, cases that could be considered as severe were not included in our study.

As it is known, the most common symptoms of COVID19 are fever, cough, dyspnea, and pneumonia characterized by bilateral infiltrates in the lung imaging $[15,16]$. There is no specific clinical feature that can reliably differentiate COVID-19 from other viral respiratory infections. In a study examining 138 patients hospitalized with COVID-19 pneumonia in Wuhan in terms of general clinical features, the most common clinical features at the onset of the disease were fever (99\%), fatigue (70\%), dry cough (59\%), anorexia (40\%), myalgia (35\%), dyspnea (3\%) and sputum production (27\%) [17]. In another study, fever was reported in almost all patients; however, about $20 \%$ of them were found to have a very low-grade fever. In subsequent studies, it is noteworthy that fever was not frequently observed as a finding $[18,19]$. The rate of patients with fever of $37^{\circ}$ and above was only $27.5 \%$ in our study.

The most obvious theory of the neurological involvement of the COVID-19 virus is through the Angiotensinconverting enzyme 2 (ACE2) mechanism. ACE2 is defined as a functional receptor for SARS CoV-2 and the virus reaches host cells in this way. This enzyme receptor is most commonly found in lung type 2 alveoli. However, it is also expressed by many cells, including glial cells and neurons. The expression and distribution of ACE2 suggests that SARS-CoV-2 can cause neurological involvement through direct or indirect mechanisms [20, 21]. In particular, it is mentioned that SARS-CoV-2 can pass through the cribriform lamina of the ethmoid bone through the ACE2 receptor, and transneuronal transportation can be achieved through the olfactory nerve. Based on this information, it seems likely that the virus can also affect the functions of other cranial nerves. In a retrospective study by Mao et al., neurological involvement or symptoms were reported in $36.4 \%$ of 214 patients (mean age $52.7 \pm 15.5$ ) with PCR positive for COVID-19. While dizziness (16.8\%) and headache (13.1\%) were the most common symptoms of the central nervous system, hypogeusia (5.6\%) and hyposmia (5.1\%) have been reported as the most common peripheral nervous system symptoms [22]. In our study, the incidence of headache and dizziness in patients was found to be remarkably higher compared to the literature.

Although there is no comprehensive study on the prevalence of otolaryngologic symptoms in the literature so far, sore throat and rhinorrhea are the most frequent complaints that have been mentioned [23]. There is an increasing number of studies, which indicate that the most common clinical findings in terms of otolaryngologic symptoms are the smell and taste dysfunction [24, 25]. In a study using a questionnaire method conducted with 59 patients diagnosed with COVID-19 in Italy, smell or taste disorder was reported in 34\%, and both smell and taste disorder in 19\% [26]. In another study evaluating whether there was a difference in taste and smell in patients with and without positive RT-PCR test, it was reported that taste and smell disorder was higher in patients with positive RT-PCR test [27]. In our study, the most common otolaryngologic symptoms were the loss of smell and taste, which is important in terms of supporting the literature data on COVID-19. Again, another important point we determined about the sense of smell and taste is that the incidence and severity of it are higher in individuals under 60 years of age. While the rate of loss of smell / taste disturbance was higher in women, the incidence of smell disturbance was higher in patients with allergic rhinitis. These data contribute to the literature on this subject.

When we look at the frequency distribution of nasal symptoms, one of the first findings of COVID-19, it is seen that the rate of complaints such as rhinorrhea is lower compared to other symptoms [28]. In this respect, COVID-19 differs from other upper respiratory viral infections [29]. In our study, the percentage of nasal obstruction was around $27 \%$, while the rate of complaints such as runny nose and itching was around $14 \%$. Another important point in terms of nasal symptoms in our study was that except for loss of smell and itching, there was no significant difference in other findings in patients with sinonasal pathology. In addition, sore throat (32\%) was found to be a common complaint in terms of pharyngeal and laryngeal symptoms. It has not been mentioned as a definite symptom in the literature; however, $19 \%$ of the patients, who participated in our study, were complaining about voice impairment.

In the literature, there is not enough data on the effect of vestibular and acoustic nerve in COVID-19 cases yet. In this respect, the most striking data in our study was that the complaint of dizziness was found at a rate of $31 \%$. The percentage of patients with tinnitus complaints was around $11 \%$. In addition the dizziness rate was more common in individuals under 60 years of age and female. In this regard, although there are no comprehensive studies in the literature, only a case of COVID-19 complaining of dizziness was reported [30]. Audiology laboratories were closed to prevent personnel contamination during the time we followedup the patients and objective tests could not be performed. These datas suggest that otological and vestibular symptoms should also be questioned in COVID-19 patients. However, large series of studies with objective tests are required for precise data.

In addition to otolaryngological symptoms, gastrointestinal findings (i.e., nausea and diarrhea) have been reported; and these may also be complaints of admission in some patients. In a systematic meta-analysis of studies reporting on gastrointestinal symptoms in patients with confirmed COVID-19, the prevalence of gastrointestinal symptoms was $18 \%$ overall; diarrhea, nausea/vomiting, or abdominal pain were reported at 13,10 and $9 \%$, respectively [31]. In 
our study, the rate of patients who mentioned having nausea/ vomiting was $31 \%$, and it was concluded that nausea/vomiting may be an important finding in terms of the distribution of symptoms as it is part of neurovegetative symptoms.

The point that we evaluated differently from the literature in our study was whether the symptoms differed in terms of variables such as age, gender, clinical status, and concominant systemic and otolaryngologic diseases. Accordingly, the point that draws attention in terms of age was that many symptoms were statistically significantly higher in the group under 60 years of age. From this point of view, in studies investigating the distribution of symptoms according to age, it was emphasized that, unlike our study, some symptoms were more common in older individuals $[32,33]$. Similarly, the fact that many symptoms are seen more frequently in women than men may be important in terms of being affected by COVID-19 infection. Again, as another data, headache was more common in patients with HT and DM. Although there is no specific information about the relationship between headache and disease in the literature, it may not be sufficient to provide precise data, since our study group is small.

When the data were examined in terms of the duration of the symptoms, the longest-onset symptoms were observed to be related to taste and smell. In a review on olfactory functions in COVID-19 cases, it was stated that it may take more than a week to replace olfactory/gustatory functions. It is seen that the data we obtained are compatible with the literature in this respect [32]. Therefore, we believe that the data in our study will be useful in terms of including the duration of symptoms.

When the symptoms were evaluated by factor analysis, similar complaints were included in the same subgroups, which was an important finding in terms of showing the validity of the data obtained in our study. Thus, otological, pharyngeal, nasal, smell, and taste, neurovegetative symptoms were determined to form subgroups among themselves. This situation was interpreted as COVID-19 affecting a certain region in the body and causing associated symptoms.

\section{Conclusion}

It is not always possible to identify patients with SARSCoV-2 infection only by clinical presentation due to the unspecific nature of the early symptoms. This is also valid for otolaryngologic manifestations and it should be known that quite different symptoms can be encountered as reported in our study. So far, the most frequently mentioned symptoms of SARS CoV-2 infection in the literature have initially been referred to as sore throat and mild cough, and later as hyposmia/anosmia or loss of taste. In particular, many studies have focused on olfactory functions. However, it was observed in our study that many otolaryngologic findings such as dizziness, headache, and even voice changes may be related to COVID-19. Therefore, focusing only on certain symptoms can create errors in the initial diagnosis and referral of patients. It is believed that further studies on these symptoms with objective evaluations would provide more data in terms of involvement and findings for the head and neck region of COVID-19.

Author contributions MÖK: conception and design of the work, collecting data, analysis, writing manuscript, final approval of manuscript, OKE: collecting data, analysis, writing manuscript, final approval of manuscript, MAÖ: collecting data, writing manuscript, final approval of manuscript, MG: collecting data, writing manuscript, final approval of manuscript.

Funding None.

Availability of data and material Yes.

\section{Compliance with ethical standards}

Conflict of interest The authors have no conflicts of interest to report.

Ethical approval The study protocol was approved by the Ethics Committee of Sakarya University Faculty of Medicine (71522473 / 050.01.04).

\section{Appendix}

Study questionnaire and clinical datas.

\section{Section 1. Personal information}

1.1. Name-Surname:

1.2. Age:

1.3. Sex:

1.4. Phone Number:

1.5. Do you have any chronic illnesses?

If yes, please note:

1.6. Do you take any medications on daily basis?

If yes, please note:

1.7. Have you had any of the following otolaryngological disorders before onset COVID-19 infection?

Prennial/seasonal allergic rhinitis:

Nasal septal deviation:

Chronic sinusitis/nasal polyposis:

Hearing loss:

Tinnitus:

Vertigo/Dizziness: 


\section{Section 2. Laboratory and clinic findings}

\subsection{COVID-PCR Test result:}

2.2. Thorax CT findings:

2.3. Laboratory findings:

2.4. General clinical findings:

Fever: Pulse: Arterial Blood Pressure:

2.3. Clinical severity:

\section{Section 3. Otolaryngological symptoms:}

Please mark if you have a new onset complaint of the following below during 19 infectious disease. Indicate how many days, if any.

Yes No Duration (days).

A. Itching / Irritation / Redness in the eyes.

B. Hearing loss/impairment in the ear.

C. Tinnitus/humming in the ear.

D. The feeling of spinning/moving around.

E. Loosing balance/dizzy.

F.Nausea/Vomiting.

G.Headache.

H.Stuffed nose.

I. Runny Nose.

J.Nasal itching/sneeze.

Please indicate the degree of hyposmia/hypogeusia on 10 scale VAS (If present).

K. Sense of smell affected.

012345678910

(10 indicate normal)

L. Sense of taste affected.

012345678910

(10 indicate normal)

M. Sore throat.

N. Swallowing difficulty.

O. Discomfort/irritation/ something stuck in the throat.

P.Voice impairment/hoarseness.

R. Shortness/difficulty of breathe.

S.Dry cough.

\section{References}

1. Guan WJ, Ni ZY, Hu Y et al (2020) Clinical characteristics of Coronavirus disease 2019 in China. N Engl J Med 382:17081720. https://doi.org/10.1056/NEJMoa2002032

2. Johns Hopkins University and Medicine (2020) Coronavirus resource center. https://coronavirus.jhu.edu/. (Accessed 16 Sept 2020).
3. Zhang X, Chen X, Chen L et al (2020) The evidence of SARSCoV-2 infection on ocular surface. Ocul Surf 18:360-362. https ://doi.org/10.1016/j.jtos.2020.03.010

4. Zaki AM, van Boheemen S, Bestebroer TM, Osterhaus AD, Fouchier RA (2012) Isolation of a novel coronavirus from a man with pneumonia in Saudi Arabia. N Engl J Med 367:1814-1820

5. Gilani S, Roditi R, Naraghi M (2020) COVID-19 and anosmia in Tehran. Iran Med Hypotheses 141:109757. https://doi. org/10.1016/j.mehy.2020.109757

6. Young BE, Ong SWX, Kalimuddin S et al (2020) Epidemiologic features and clinical course of patients infected with SARS-CoV-2 in Singapore. JAMA 323:1488-1494. https://doi. org/10.1001/jama.2020.3204

7. Wan S, Xiang Y, Fang W, Zheng Y et al (2020) Clinical features and treatment of COVID-19 patients in Northeast Chongqing. J Med Virol 92:797-806. https://doi.org/10.1002/jmv.25783

8. Roland LT, Gurrola JG 2nd, Loftus PA, Cheung SW, Chang JL (2020) Smell and taste symptom-based predictive model for COVID-19 diagnosis. Int Forum Allergy Rhinol 10:832-838. https://doi.org/10.1002/alr.22602

9. Soler Z, Patel Z, Turner J, Holbrook E (2020) A primer on viralassociated olfactory loss in the era of COVID-19. Int Forum Allergy Rhinol 10:814-820. https://doi.org/10.1002/alr.22578

10. Lechien JR, Chiesa-Estomba CM, Place S et al (2020) Clinical and epidemiological characteristics of 1420 European patients with mild-to-moderate Coronavirus Disease 2019. J Intern Med. https://doi.org/10.1111/joim.1308

11. World Health Organization (2020) Report of the WHO-China Joint Mission' on Coronavirus 358 Disease 2019 (COVID-19). World Health Organization, Geneva

12. American Academy of Otolaryngology Head 455 and Neck Surgery (AAO-HNS): Anosmia Reporting tool (2020). https:// www.entnet.org/content/reporting-tool-patients-anosmia-relat ed-covid-19. (Accessed 20 Apr 2020)

13. Gao X, Yuan Z, Yang Di et al (2020) A family cluster of severe acute respiratory syndrome coronavirus 2 infections. Eur J Clin Microbiol Infect Dis 8:1-5. https://doi.org/10.1007/s10096-02003880-1

14. Singhal TA (2020) Review of Coronavirus Disease(COVID19)-2019. Indian J Pediat 87:281-286. https://doi. org/10.1007/s12098-020-03263-6

15. Adhikari SP, Meng S, Wu YJ et al (2020) Epidemiology, causes, clinical manifestation and diagnosis, prevention and control of coronavirus disease (COVID-19) during the early outbreak period: a scoping review. Infect Dis Poverty 9:29. https://doi. org/10.1186/s40249-020-00646-X

16. Liu Y, Yan LM, Wan L et al (2020) Viral dynamics in mild and severe cases of COVID-19. Lancet Infect Dis 20:656-657. https ://doi.org/10.1016/S1473-3099(20)30232-2

17. Wang D, Hu B, Hu C et al (2020) Clinical characteristics of 138 hospitalized patients with 2019 novel coronavirus-infected pneumonia in Wuhan, China. JAMA 323:1061-1069. https:// doi.org/10.1001/jama.2020.1585

18. Zhou M, Zhang X, Qu J (2020) Coronavirus disease 2019 (COVID-19): a clinical update. Front Med 2:1-10. https://doi. org/10.1007/s11684-020-0767-8

19. Chen N, Zhou M, Dong X et al (2020) Epidemiological and clinical characteristics of 99 cases of 2019 novel coronavirus pneumonia in Wuhan, China: a descriptive study. Lancet 395:507-513. https://doi.org/10.1016/S0140-6736(20)30211-7

20. Baig AM, Khaleeq A, Ali U, Syeda H (2020) Evidence of the COVID-19 virus targeting the CNS: tissue distribution, hostvirus interaction, and proposed neurotropic mechanisms. ACS Chem Neurosci 11:995-998. https://doi.org/10.1021/acsch emneuro.0c00122 
21. Wang L, Shen Y, Li M, Chuang H et al (2020) Clinical manifestations and evidence of neurological involvement in 2019 novel coronavirus SARS-CoV-2: a systematic review and meta-analysis. J Neurol. https://doi.org/10.1007/s00415-020-09974-2

22. Mao L, Jin H, Wang M et al (2020) Neurological Manifestations of Hospitalized Patients with COVID-19 in Wuhan, China: a retrospective case series study. JAMA Neurol 77:1-9. https://doi. org/10.1001/jamaneurol.2020.1127

23. Meng X, Deng Y, Dai Z, Meng Z (2020) COVID-19 and anosmia: a review based on up-to-date knowledge. Am J Otolaryngol 41:102581. https://doi.org/10.1016/j.amjoto.2020.102581

24. Gautier JF, Ravussin Y (2020) A new symptom of COVID-19: loss of taste and smell. Obesity (Silver Spring) 28:848-852. https ://doi.org/10.1002/oby.22809352

25. Agyeman AA, Chin KL, Landersdorfer KB, Liew D, Ofori-Asenso $\mathrm{R}$ (2020) Smell and taste dysfunction in patients with COVID-19: a systematic review and meta-analysis. Mayo Clin Proc 95:16211631. https://doi.org/10.1016/j.mayocp.2020.05.030

26. Vaira LA, Salzano G, Deiana G, De Riu G (2020) Anosmia and ageusia: common findings in 384 COVID-19 patients. Laryngoscope 130:1787. https://doi.org/10.1002/lary.28692

27. Sayin İ, Yaşar KK, Yazici ZM (2020) Taste and smell impairment in COVID-19: An AAO-HNS anosmia reporting tool-based comparative study. Otolaryngol Head Neck Surg. https://doi. org/10.1177/0194599820931820

28. Heikkinen T, Järvinen A (2003) The common cold. Lancet 361:51-59. https://doi.org/10.1016/S0140-6736(03)12162-9
29. Krajewska J, Krajewski W, Zub K, Zatoński T (2020) COVID-19 in otolaryngologist practice: a review of current knowledge. Eur Arch Otorhinolaryngol 277:1885-1897. https://doi.org/10.1007/ s00405-020-05968-y

30. Kong Z, Wang J, Li T, Zhang Z, Jian J (2020) 2019 novel coronavirus pneumonia with onset of dizziness: a case report. Ann Transl Med 8:506-509. https://doi.org/10.21037/atm.2020.03.89

31. Cheung KS, Hung IFN, Chan PPY et al (2020) Gastrointestinal manifestations of SARS-CoV-2 infection and virus load in fecal samples from the hong kong cohort and systematic review and meta-analysis. Gastroenterology 159:81-95. https://doi. org/10.1053/j.gastro.2020.03.065

32. Costa KVTD, Carnaúba ATL, Rocha KW et al (2020) Olfactory and taste disorders in COVID-19: a systematic review. Braz J Otorhinolaryngol S1808-8694(20):30066-30075. https://doi. org/10.1016/j.bjorl.2020.05.008

33. Yang Y, Yang M, Shen C et al (2020) Evaluating the accuracy of different respiratory specimens in the laboratory diagnosis and monitoring the viral shedding of $2019-\mathrm{nCoV}$ infections. Medrxiv. https://doi.org/10.1101/2020.02.11.20021493

Publisher's Note Springer Nature remains neutral with regard to jurisdictional claims in published maps and institutional affiliations. 